\title{
High-Resolution Vessel Wall Magnetic Resonance Imaging of Varicella-Zoster Virus Vasculopathy Affecting the Vertebrobasilar Artery
}

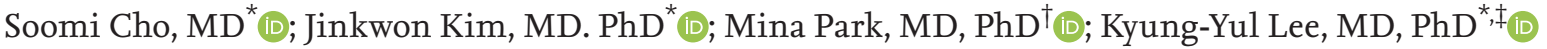 \\ Department of Neurology, Gangnam Severance Hospital, Yonsei University College of Medicine*, Seoul; Department of Radiology, Gangnam \\ Severance Hospital, Yonsei University College of Medicine ${ }^{\dagger}$, Seoul; Severance Institute for Vascular and Metabolic Research, Yonsei University \\ College of Medicine ${ }^{\ddagger}$, Seoul, Korea
}

Varicella-zoster virus (VZV) has been known to cause intracranial vasculopathy. Herein, we report the case of a patient with VZV vasculopathy involving the vertebrobasilar system who underwent serial high-resolution vessel wall magnetic resonance imaging (HRVW-MRI). The disease continued to worsen radiographically despite clinical symptom improvement. We suggest that VZV vasculopathy may exhibit clinicoradiological dissociation that requires ongoing medical attention. HRVW-MRI may play a critical role in monitoring the disease activity.

J Neurosonol Neuroimag 2020;12(1):51-54

Key Words: Varicella zoster virus infection; Vascular diseases; Magnetic resonance imaging
Received: January 23, 2020

Revised: February 19, 2020

Accepted: March 2, 2020

Correspondence:

Kyung-Yul Lee, MD, PhD

Department of Neurology, Gangnam Severance Hospital, Yonsei University College of

Medicine, 211 Eonju-ro,

Gangnam-gu, Seoul 06273, Korea

Tel: $+82-2-2019-3325$

Fax: $+82-2-3462-5904$

E-mail:kylee@yuhs.ac
Varicella-zoster virus (VZV) has been known to cause intracranial vasculopathy, which can result in various cerebrovascular complications such as transient ischemic attack, ischemic and hemorrhagic stroke, aneurysm, and arterial dissection. ${ }^{1,2}$ Previous studies indicated that the anterior circulation, especially the distal internal carotid or proximal middle cerebral arteries, is frequently affected in VZV vasculopathy. ${ }^{3}$ We report a case of VZV vasculopathy involving the vertebrobasilar system, which was diagnosed and followed up with high-resolution vessel wall magnetic resonance imaging (HRVW-MRI).

\section{CASE}

A 51-year-old man was admitted with sudden left-sided hemiparesis and dysarthria. He reported no history of chronic illnesses, including cancer or autoimmune diseases. Six weeks prior to the symptoms, he had visited the emergency department with severe headache and a painful blistering rash behind his right ear and posterior neck, leading to a diagnosis of herpes zoster and a 7-day prescription of oral valacyclovir.

The initial neurological examination revealed left central-type facial palsy and a pronator drift in the left arm. His left leg weakness was mild, and he could walk without difficulty. The National Institutes of Health Stroke Scale score was 3.

Brain magnetic resonance (MR) imaging revealed an acute right pontine infarction on the diffusion-weighted image (Fig. 1A). The susceptibility-weighted image showed a subarachnoid hemorrhage in the prepontine cistern (Fig. 1B, C) and a minimal wall hemorrhage in the right distal vertebral artery (VA). MR angiography revealed severe stenosis of the right distal VA (Fig. 1D). VA dissection was first suspected, and HRVW-MRI was performed to confirm the arterial wall pathology.

HRVW-MRI taken 2 days after admission demonstrated severely tapered right distal VA with concentric wall thickening and enhancement, which was strongly suggestive of vasculitis (Fig. $1 \mathrm{E}$ ). The basilar artery (BA) was patent without any enhancement (Fig. $1 F$ ). Given that the vessel wall inflammation may be a pathogenetic factor of arterial dissection, ${ }^{4}$ vasculitis was concluded to be the primary cause of VA dissection. 

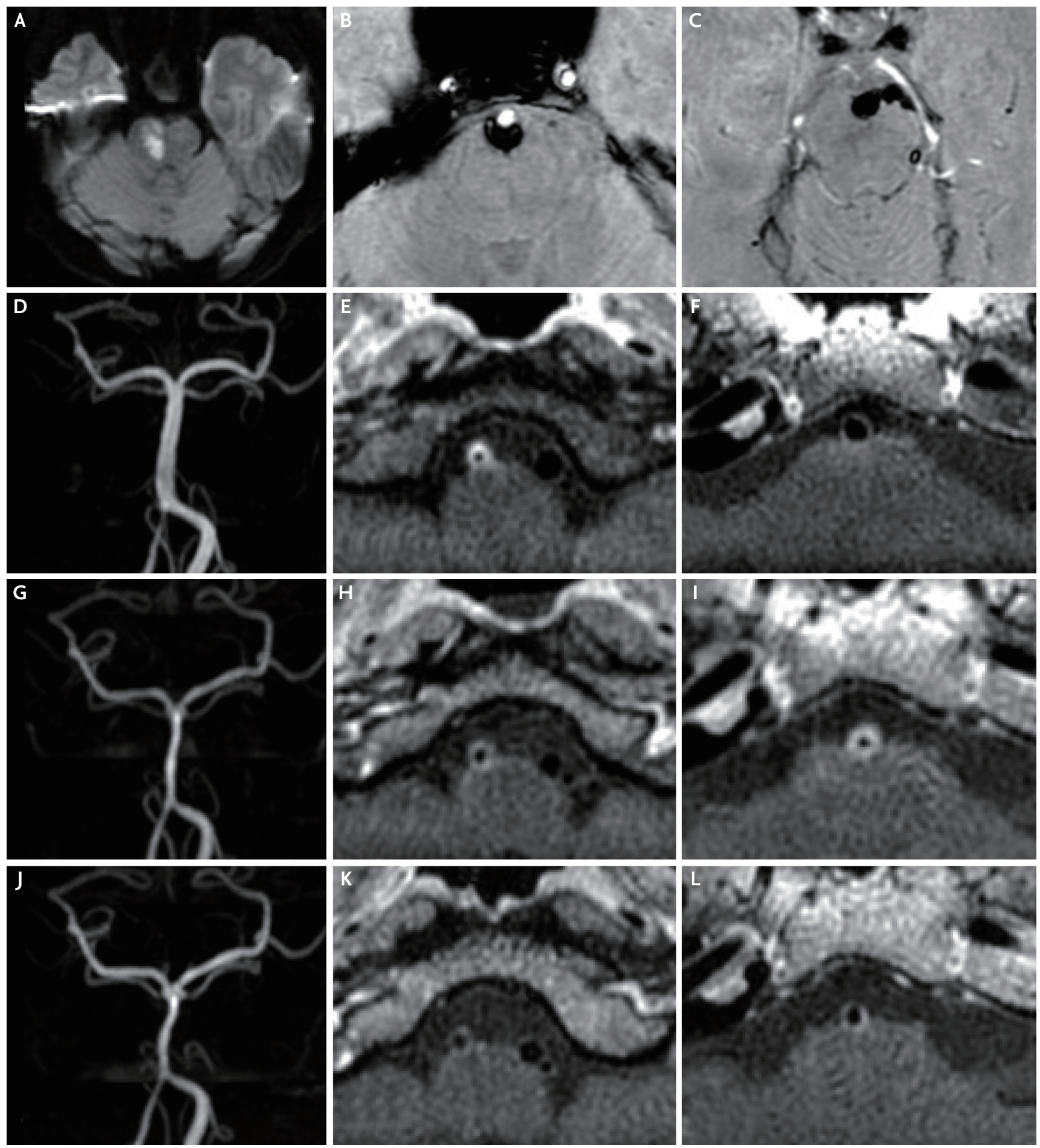

FIG. 1. Brain magnetic resonance imaging, magnetic resonance angiography (MRA), and vessel wall imaging scans of the present patient. (A-F) Initial images. (A) The diffusion-weighted image shows an acute right pontine infarction. (B, C) The susceptibility-weighted images demonstrate subarachnoid hemorrhage in the prepontine cistern, originating from trace amounts of hemorrhage in the wall of the right distal vertebral artery (VA). (D) The MRA image shows a severe stenosis of the right distal VA. (E, F) The vessel wall images demonstrate concentric wall thickening and enhancement of the right distal VA, and a normal basilar artery (BA). (G-I) Two-month follow-up images. (G) The MRA image shows improvement of the stenosis of the right distal VA and a newly developed diffuse luminal narrowing of the BA. $(\mathrm{H}, \mathrm{I})$ The vessel wall images show a reduction in the enhancement of the right distal VA but a newly developed, diffuse, concentric wall thickening and enhancement of the BA. (J-L) Five-month follow-up images. (J) The MRA image demonstrates an insignificant change in the stenosis of the vertebrobasilar arteries. (K, L) The vessel wall images show a reduction in the extent of the enhancement of the right distal VA and BA. 
Laboratory tests were performed to identify various causes of cerebral vasculitis. No abnormalities were observed in the complete blood count, routine chemistry tests, and urinalysis, indicating a lack of evidence of systemic organ involvement. The results of the antinuclear antibody (ANA), perinuclear antineutrophil cytoplasmic antibody (p-ANCA), and cytoplasmic antineutrophil cytoplasmic antibody (c-ANCA) were all negative. The erythrocyte sedimentation rate was $24 \mathrm{~mm}$ /hour, which showed a slight increase from the normal values of o-15 mm/hour, while the C-reactive protein level was within its normal range.

Regarding the possibility of VZV vasculopathy occurring up to 6 months after onset of zoster infection ${ }^{1}$ and excluding other possible causes, VZV vasculopathy was found to be the most likely cause of the vasculitis. The patient received oral prednisolone $30 \mathrm{mg} /$ day in combination with antiplatelets. Mild dysarthria was the only sequelae upon discharge.

Two months later, the patient did not present any neurological symptoms. However, follow-up HRVWMRI revealed a newly developed, diffuse, concentric wall thickening and enhancement of the BA with diffuse luminal narrowing (Fig. 1G-I). We restarted the oral valacyclovir and steroid therapies because an active vascular inflammation involving the BA was seen. A third HRVW-MRI, obtained 5 months after the initial stroke, demonstrated a decrease in the extent of the enhancement in the right distal VA and BA, and overall improvement of vessel wall inflammation and stenosis (Fig. 1J-L).

\section{DISCUSSION}

The present case demonstrates that VZV vasculopathy may also occur in the vertebrobasilar arterial system. Direct VZV invasion of cerebral arteries, which leads to inflammation and pathological vascular remodeling, is thought to be the mechanism of VZV vasculopathy. ${ }^{5}$ Thickened intima, disrupted internal elastic lamina, and loss of smooth muscle cells were revealed in the histological and immunohistochemical studies of VZV-infected arteries. ${ }^{5}$

Diagnosis of VZV vasculopathy is not straightforward, as stroke may occur months to years after zoster infec- tion $^{6}$ and other risk factors of stroke may coexist. In the study of 19 patients with zoster rash, the mean interval from rash to neurological symptoms or signs was 4.1 months, and the interval ranged from the same day to 2.5 years. ${ }^{6}$ Careful history taking, including recent herpes zoster infection, can aid early diagnosis. Obtaining cerebrospinal fluid for the detection of anti-VZV antibodies or VZV DNA is recommended to confirm the diagnosis. ${ }^{1}$ However, negative virological findings do not always exclude the diagnosis. Reasonable suspicion is of importance, and HRVW-MRI may play a critical role in diagnosing VZV vasculopathy. HRVW-MRI is recognized for its usefulness in differentiating various intracranial arterial pathologies such as intracranial atherosclerosis, vasculitis, dissection, and reversible cerebral vasoconstriction syndrome. ${ }^{7}$ Imaging findings of VZV vasculopathy usually show focal or diffuse areas of stenosis in MR angiography or cerebral angiography, with corresponding concentric thickening and contrast uptake in HRVW-MRI. ${ }^{3}$

The lack of evidence, strategies for follow-up assessment, and treatments for VZV vasculopathy still remain elusive. Despite the clinical symptom improvement, VZV vasculopathy may continue to worsen radiographically as presented in this study. The patient showed clinical and radiographic improvements with oral prednisolone therapy, although the steroid therapy was once discontinued but was then resumed after confirmation of findings suggestive of ongoing disease on follow-up HRVW-MRI. The efficacy of prolonged use of steroids for VZV vasculopathy is still in question, but treatment with intravenous acyclovir therapy is recognized for stabilizing and improving vasculopathy. ${ }^{1,5}$ Given that VZV vasculopathy is potentially treatable $\mathrm{e}^{5}$ and may exhibit clinicoradiological dissociation that requires ongoing medical attention, we recommend that follow-up HRVW-MRI should be considered regardless of clinical symptoms to monitor the disease activity. However, whether the treatment duration should be based on clinical symptoms or imaging findings still remains controversial, necessitating further investigations.

\section{Conflicts of Interest}

No potential conflicts of interest relevant to this article was reported. 


\section{REFERENCES}

1. Gilden D, Cohrs RJ, Mahalingam R, Nagel MA. Varicella zoster virus vasculopathies: diverse clinical manifestations, laboratory features, pathogenesis, and treatment. Lancet Neurol. 2009;8:731-740.

2. Nagel MA, Gilden D. Varicella zoster virus vasculopathy. In: Caplan LR, Biller J. Uncommon causes of stroke. 3rd ed. Cambridge: Cambridge University Press. 2018;71-76.

3. Cheng-Ching E, Jones S, Hui FK, Man S, Gilden D, Bhimraj A, et al. High-resolution MRI vessel wall imaging in varicella zoster virus vasculopathy. J Neurol Sci. 2015;351:168-173.

4. Pfefferkorn T, Saam T, Rominger A, Habs M, Gerdes LA, Schmidt C, et al. Vessel wall inflammation in spontaneous cervical artery dissection: a prospective, observational positron emission tomography, computed tomography, and magnetic resonance imaging study. Stroke. 2011;42:1563-1568.

5. Nagel MA. Varicella zoster virus vasculopathy: clinical features and pathogenesis. J Neurovirol. 2014;20:157-163.

6. Nagel MA, Cohrs RJ, Mahalingam R, Wellish MC, Forghani $\mathrm{B}$, Schiller A, et al. The varicella zoster virus vasculopathies: clinical, CSF, imaging, and virologic features. Neurology. 2008;70:853-860.

7. Mandell DM, Mossa-Basha M, Qiao Y, Hess CP, Hui F, Matouk $\mathrm{C}$, et al. Intracranial vessel wall MRI: principles and expert consensus recommendations of the American Society of Neuroradiology. AJNR Am J Neuroradiol. 2017;38:218229. 